\title{
A hybrid model of cell migration in zebrafish embryogenesis
}

\author{
Ezio Di Costanzo ${ }^{1, a}$, Roberto Natalini ${ }^{2, b}$, and Luigi Preziosi ${ }^{3, c}$ \\ ${ }^{1}$ Istituto per le Applicazioni del Calcolo "M. Picone" - Consiglio Nazionale delle Ricerche, Via Pietro \\ Castellino 111, 00131 Naples, Italy. \\ ${ }^{2}$ Istituto per le Applicazioni del Calcolo "M. Picone" - Consiglio Nazionale delle Ricerche, Via dei Taurini 19, \\ 00185 Rome, Italy. \\ ${ }^{3}$ Department of Mathematical Sciences, Politecnico di Torino, Corso Duca degli Abruzzi 24, 10129 Turin, \\ Italy.
}

\begin{abstract}
Starting from the results of recent biological experiments, we propose a discrete in continuous mathematical model for the morphogenesis of the posterior lateral line system in zebrafish. Our hybrid description is discrete on the cellular level and continuous on the molecular level. We prove the existence of steady solutions corresponding to the formation of particular biological structures, the neuromasts. Numerical simulations are performed to show the dynamics of the model and its accuracy to describe the evolution of the cell aggregate by a qualitative and quantitative point of view. Some related models, applied to the collective motion of cells, and to the behaviour of cardiac stem cells, are indicated.
\end{abstract}

\section{Introduction}

The study in the field of collective motion, plays an essential role in a number of real-life situations. In particular, the research in the collective motion of cells brings important advantages understanding growth, development and disease of many organs and tissues. Some species, especially those that can be easily treated experimentally, represent a paradigm for understanding more complex organisms, including humans. For this, in recent year collective dynamics has been extensively studied in a great variety of cell systems [1]. In [2], starting from the results obtained in recent biological experiments [3-5], we have have proposed a hybrid mathematical model describing self-organizing cell migration in the morphogenesis of the posterior lateral line system (PLL) in the model organism of zebrafish (Danio rerio). The great interest for this sensory system and the need to formulate new mathematical theoretical models is motivated by its important role in the current scientific research, and by the still not complete understanding of the general mechanism for cells arranging and organization [5]. The lateral line is a fundamental sensory system, present in fish and amphibians, that extends from the head to the tail along each flank of the individual. It is involved in a large variety of behaviours: e.g. detect movement and vibrations in the surrounding water, prey and predator detection. The main sensory

\footnotetext{
ae-mail: e.dicostanzo@na.iac.cnr.it

be-mail: roberto.natalini@cnr.it

ce-mail: luigi.preziosi@polito.it
} 
organs of the lateral line are the neuromasts, located on the body surface in specific patterns: a rosetteshaped structure with one or two cells in the centre (leader cells) innervated by sensory neurons and surrounding by other support cells (follower cells). The function of the lateral line represents a subject of great interest in biology. Its study has more deeply explained many general issues: interactions between multiple signalling and coordination of cell during development, behaviour of collective motion of cells, response of an organ to injury and the healing of damaged components. In particular, zebrafishes represent useful vertebrate model organisms whose genome is fully sequenced. Great advantages are due to their regenerative abilities, to robustness and transparency of the embryos. The zebrafish model has been widely tested in many fields of biology: developmental biology, oncology, genetics, stem cell and regenerative medicine.

\section{Biological observations}

Experimental evidences on the zebrafish PLL have shown an initial elongated group of mesenchymal cells (called primordium), in the range 80-100 cells, located in the otic vesicle. In few hours postfertilization a total migration of the primordium begins from the head to the tail of the embryo at an estimated velocity $\left(\approx 69 \mu \mathrm{m} \mathrm{h}^{-1}\right)$ [5]. Subsequently, a cell transition, occurring in the rear of the migrating group, makes some cells epithelial-like (mesenchymal-epithelial transition) and rosette-shaped structures (proto-neuromasts) begin to emerge. When these cell aggregates become fully mature, they are detached and deposited along the body of the embryo, giving rise to the growth and location of neuromasts [4]. In general we can split the morphogenesis of the zebrafish lateral line primordium in two primary mechanisms: a collective migration, and a neuromast assembly. The first process is regulated by a haptotactic chemokine, stromal cell-derived factor-1 a (SDF-1a), expressed by the substratum and detected by the primordium [3]. Moreover, the total migration is ensured by a series of cell-cell interactions via mechanical forces, and by a cell-substratum adhesion. The neuromast formation, in the second stage, is regulated by two main chemotactic factors expressed by the primordium, the equivalent fibroblast growth factors FGF3-FGF10, and by their receptors FGFRs. FGF signal and its receptor are mutually exclusive: FGF3-FGF10 are broadly expressed in the leading region of the primordium and focused in one or two cells in the centre of the rosettes in the trailing region, on the other hand FGFR is expressed in the trailing region except the FGF sources [5]. This let us divide the cell population in two kinds: leader cells that produce FGF signal and follower cells that, activating the receptor FGFR, do not produce any signal. At the beginning, the primordium contains only leader cells, afterwards, a process of differentiation on the backside makes some cells follower-like, except one or two cells located in the centre. The follower cells are then driven toward the FGF sources to form rosette-shaped aggregates (the future neuromasts), that subsequently are detached during the migration. Hence a new rosette is generated in a cyclic process. Finally, the leader-to-follower transition in the trailing region can be represented by three conditions: a low level of SDF-1a, a high level of FGF and an influence of lateral inhibition [5]. This last condition can be read here as follows: a cell that adopts the follower fate inhibits its immediate neighbouring cells from doing likewise. In this way a central leader will be more inhibited to become follower, while a cell at the edge of the primordium will be more likely to undergo the transition. See [2] for a more complete description.

\section{The mathematical model}

Taking into account the low number of cells involved, our mathematical approach for the above discussed morphogenesis process adopts a hybrid discrete in continuous description: cells are treated as discrete entities moving in a continuous space, and chemical signals, at molecular level, are described by continuous variables. On the chemical scale we adopt diffusion and chemotaxis equations, while 
on the cellular scale typical effects of collective dynamics will be taken into account [1]. Although the cell are discrete points, we recover their shape through suitable detection radii. Moreover, we can consider the 2D case due to the flattened morphology of the primordium. Our general aim is to obtain a minimal model which is able to describe the total cell migration, the formation and the detachment of the neuromasts, in the spatial and temporal scale of the experimental observations. Secondly, we require the existence and stability of the rosette structures characterizing the neuromasts, as stationary solutions of the system. This provides possible restrictions on the range of some parameter of the model. Other parameters, used in the numerical simulations, are obtained from biological literature or by a numerical calibration. Let $\mathbf{X}_{i}(t)$ be the position of the $i$-th cell, $\varphi_{i}(t)$ the state variable assuming the values 0-1 respectively for a follower-leader cell, $f(\mathbf{x}, t)$ the concentration of the FGF (equivalent FGF3-FGF10) and $s(\mathbf{x}, t)$ the concentration of the chemokine SDF-1a, our model reads:

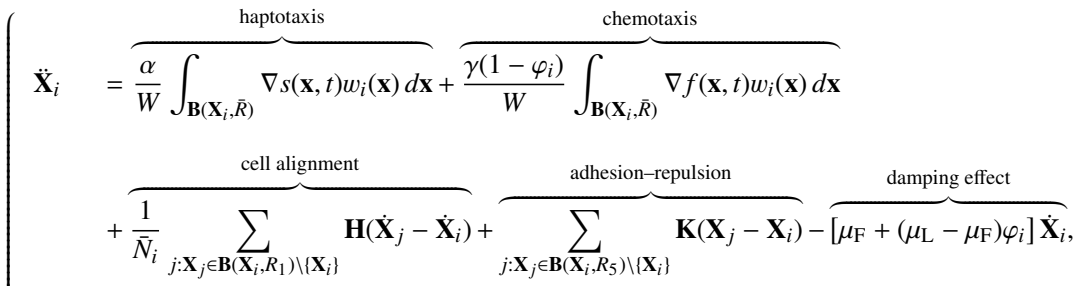

$$
\begin{aligned}
& \varphi_{i}=\left\{\begin{aligned}
0, & \text { if } \overbrace{\frac{\delta}{W} \int_{\mathbf{B}\left(\mathbf{x}_{i}, \bar{R}\right)} s(\mathbf{x}, t) w_{i}(\mathbf{x}) d \mathbf{x}}^{\text {SDF-1a concentration }}-\overbrace{\frac{k_{\mathrm{F}}+\left(k_{\mathrm{L}}-k_{\mathrm{F}}\right) \varphi_{i}}{W} \int_{\mathbf{B}\left(\mathbf{x}_{i}, \bar{R}\right)} \frac{f(\mathbf{x}, t)}{f_{\mathrm{max}}+f(\mathbf{x}, t)} w_{i}(\mathbf{x}) d \mathbf{x}}^{\text {FGF concentration }} \overbrace{\lambda \Gamma\left(n_{i}\right)}^{\text {lateral inhibition }} \leq 0, \\
1, & \text { otherwise, }
\end{aligned}\right. \\
& \partial_{t} f=\overbrace{D \Delta f}^{\text {diffusion }}+\overbrace{\xi \sum_{j=1}^{N_{\text {tot }}} \varphi_{j} \chi \mathbf{B}\left(\mathbf{x}_{j}, R_{3}\right)}^{\text {production }}-\overbrace{\eta f}^{\text {molecular degradation }}, \\
& \partial_{t} s=-\sigma s \overbrace{\sum_{j=1}^{N_{\text {tot }}} \chi \mathbf{B}_{\mathbf{B}\left(\mathbf{X}_{j}, R_{3}\right)}}^{\text {degradation }}
\end{aligned}
$$

where $\alpha, \gamma, \mu_{\mathrm{L}}, \mu_{\mathrm{F}}, \delta, k_{\mathrm{L}}, k_{\mathrm{F}}, \lambda, D, \xi, \eta, \sigma$ are given positive constants, and $\bar{R}, R_{i}, i=1, \ldots, 5$, are suitable sensing radii (some of them included in the functions $\mathbf{K}\left(\mathbf{X}_{j}-\mathbf{X}_{i}\right)$ and $\Gamma\left(n_{i}\right)$ ). More details can be found in [2]. Here we remark that some parameters can have different values depending on the leader-follower cell state ("L" and "F" markers in subscript position). In particular, the integral terms in $(1)_{1,2}$, related to the detection of a chemical signal by the $i$-th cell in its neighbourhood, represent non local effects with a weighted average, through a truncated Gaussian weight $w_{i}(\mathbf{x})$, over a ball $\mathbf{B}\left(\mathbf{X}_{i}, \bar{R}\right)$ centred in $\mathbf{X}_{i}$ and sensing radius $\bar{R}$. While first and second terms in equation (1) translate the haptotactic attraction by the SDF-1a signal and the chemotactic effect of the FGF on a follower cell $\left(\varphi_{i}=0\right)$, third and fourth addends include cell-cell interaction via mechanical forces, due to filopodia. In particular, $\mathbf{H}\left(\dot{\mathbf{X}}_{j}-\dot{\mathbf{X}}_{i}\right)$ is an alignment term inspired by the Cucker-Smale mechanism [6], though in our case it has a truncated effect (on $\bar{N}_{i}$ neighbouring cells) and it is coupled with other effects. Namely, we choose

$$
\mathbf{H}\left(\dot{\mathbf{X}}_{j}-\dot{\mathbf{X}}_{i}\right):=\left[\beta_{\mathrm{F}}+\left(\beta_{\mathrm{L}}-\beta_{\mathrm{F}}\right) \varphi_{i} \varphi_{j}\right] \frac{R_{1}^{2}}{R_{1}^{2}+\left\|\mathbf{X}_{j}-\mathbf{X}_{i}\right\|^{2}}\left(\dot{\mathbf{X}}_{j}-\dot{\mathbf{X}}_{i}\right),
$$


where $\beta_{\mathrm{F}}$ and $\beta_{\mathrm{L}}$ are positive constants. The function $\mathbf{K}\left(\mathbf{X}_{j}-\mathbf{X}_{i}\right)$ includes adhesion-repulsion effects on suitable sensing regions:

$$
\mathbf{K}\left(\mathbf{X}_{j}-\mathbf{X}_{i}\right):= \begin{cases}-\omega_{\text {rep }}\left(\frac{1}{\left\|\mathbf{X}_{j}-\mathbf{X}_{i}\right\|}-\frac{1}{R_{4}}\right) \frac{\mathbf{X}_{j}-\mathbf{X}_{i}}{\left\|\mathbf{X}_{j}-\mathbf{X}_{i}\right\|}, & \text { if } \quad\left\|\mathbf{X}_{j}-\mathbf{X}_{i}\right\| \leq R_{4} ; \\ \bar{\omega}_{\text {adh }}\left(\left\|\mathbf{X}_{j}-\mathbf{X}_{i}\right\|-R_{4}\right) \frac{\mathbf{X}_{j}-\mathbf{X}_{i}}{\left\|\mathbf{X}_{j}-\mathbf{X}_{i}\right\|}, & \text { if } \quad R_{4}<\left\|\mathbf{X}_{j}-\mathbf{X}_{i}\right\| \leq R_{5} ;\end{cases}
$$

where $R_{5}>R_{4}$ and $\omega_{\text {rep }}, \bar{\omega}_{\text {adh }}$ are differentiated constants for a leader or a follower cell. Similar terms can be found, for instance, in [7]. The last term in $(1)_{1}$ is due to the cell adhesion to the substrate, possibly with a different damping coefficient. Switch variable $\varphi_{i}$ in equation $(1)_{2}$ represents the follower-leader state and it depends on a threshold function that takes into account the three transition rules discussed in Section 2. A function $\Gamma\left(n_{i}\right)$, depending on the number of neighbouring cell, represents a simple way to translate the lateral inhibition effect, although other models can be imagined. Finally, chemical signals in equations $(1)_{3,4}$ are described using reaction-diffusion equation, with possible sources and degradation terms. Initial and boundary conditions concern initial position and cell velocity $\left(\dot{\mathbf{X}}_{i}(0)=\mathbf{0}\right)$, zero initial concentration of FGF, homogeneous Neumann boundary condition, and SDF-1a assumed distributed on a thin stripe.

\section{Main results: steady states and dynamical simulations}

In [2], starting from system (1), we have proved the existence of stationary solutions biologically relevant and consistent with the formation of the neuromast structures. Namely, we have defined particular configurations, named $N$-rosettes, formed by a leader cell in the centre, surrounded by $N$ follower cells, whose centres are located on the vertices of a regular polygon of $N$ sides, centred in the leader cell. Assuming some physically reasonable hypotheses on the distances between the cells and on the interaction radii, from geometrical considerations some properties have been deduced for our steady solutions. Among these, for instance, we will prove the existence of an upper bound for the number of cells in a $\mathrm{N}$-rosette. Such values are in a good agreement with the experimental observations shown in [5]. Moreover, the stationary model imposes important restrictions on some parameters of the system, which have been used in the numerical tests of the dynamical case. About the stability of the steady configurations we have given then a numerical assessment. In the temporal range of the real phenomenon we have observed the $N$-rosettes be stable. Finally, from a dynamical point of view some numerical simulations have been performed to show the behaviour of the model and its qualitative and quantitative accuracy to describe the evolution of the cell aggregate. The parameters of the system, fixed in part by the steady model, have been estimated from the biological-modelling literature or by a suitable numerical data fitting. Our numerical tests has shown a nice agreement with biological evidences, both on the spatio-temporal scale and on the chemical concentration scale of the physical process (Figure 1). Moreover, our model could reproduce the mechanism of self-generation of chemical gradient by the tip cells of the primordium: the total cell migration can be ensured even with an initial constant chemokine distribution along the longitudinal axis. This effect, hypothesized for the cell migration in embryos, has been recently proved in vivo on the zebrafish by [8].

\section{Conclusion and perspectives}

Following the framework of the studies [3-5] our discrete in continuous mathematical model is able to describe the main features of the considered morphogenesis process. Although the model presents 


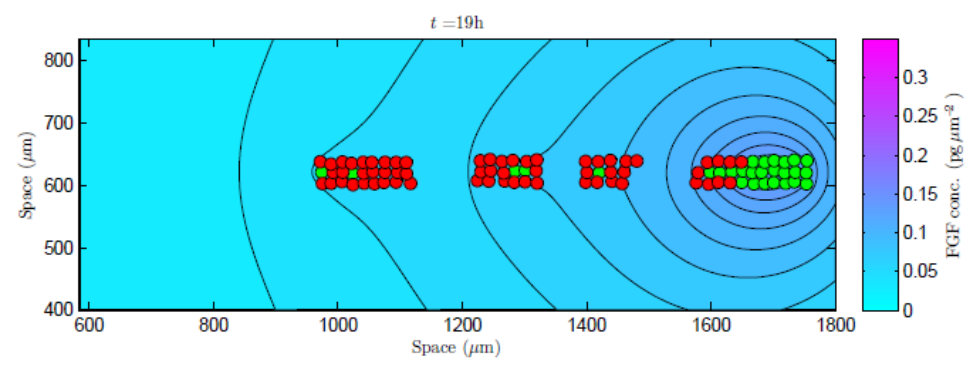

Figure 1. A numerical simulation of system (1), showing the morphogenesis of the zebrafish lateral line primordium at $t=19 \mathrm{~h}$. Green and red colours mark respectively leader and follower cells. Contour plot in the background indicates the FGF concentration, while the SDF-1a variable is not shown. In the time of observation we obtain the detachment of three neuromasts with an estimated migration velocity of about $70 \mu \mathrm{m} / \mathrm{h}$, from left to right.

some limits, as the equally circular cell shapes, and only a limited number of biological interactions have been modelled, a nice agreement with the experimental data can be inferred. Moreover, we remark that with respect to the existing biological literature, some mechanism are immediately evident, as chemotaxis, cell adhesion-repulsion, friction, diffusion, while others, as the lateral inhibition term, cell alignment, change of parameters in the leader-follower transition, have been introduced to obtain a good concordance with the observations. It would be interesting to have experimental evidence in this regard. Finally, in the recent paper [9], starting from [2], in which the Cucker-Smale model [6] is coupled with many other effects, we have proposed a hybrid model of collective motion under alignment and chemotaxis effect. This model has been studied both from an analytical and a numerical point of view, proving some time-asymptotic convergence results. In another recent paper [10], in collaboration with some clinicians, we have then applied our basic model to describe particular cell aggregates in analogous systems, as the human cardiac stem cells. The modelling of particular cell structures, the cardiospheres, has brought also improvements and extensions to our previous model.

\section{References}

[1] E. Méhes, T. Vicsek, Integr Biol 6, 831 (2014)

[2] E. Di Costanzo, R. Natalini, L. Preziosi, J of Math Biol 71, 171 (2015)

[3] P. Haas, D. Gilmour, Dev Cell 10, 673 (2006)

[4] A. Nechiporuk, D. Raible, Science 320, 1774 (2008)

[5] V. Lecaudey, G.C. Akdogan, W.H.J. Norton, D. Gilmour, Development 135, 2695 (2008)

[6] F. Cucker, S. Smale, Ieee T Automat Contr 52, 852 (2007)

[7] M.R. D’Orsogna, Y.L. Chuang, A.L. Bertozzi, L.S. Chayes, Phys Rev Lett 96, 104302 (2006)

[8] E. Donà, J.D. Barry, G. Valentin, C. Quirin, A. Khmelinskii, A. Kunze, S. Durdu, L.R. Newton, A. Fernandez-Minan, W. Huber et al., Nature 503, 285 (2013)

[9] E. Di Costanzo, R. Natalini, preprint, http://arxiv.org/abs/1507. 02980

[10] E. Di Costanzo, A. Giacomello, E. Messina, R. Natalini, G. Pontrelli, F. Rossi, M. Twarogowska, in preparation 